\title{
Application for calculation of mean aerodynamic chord of arbitrary wing planform
}

Tomáš Vogeltanz

Citation: AIP Conference Proceedings 1738, 120018 (2016); doi: 10.1063/1.4951901

View online: http://dx.doi.org/10.1063/1.4951901

View Table of Contents: http://aip.scitation.org/toc/apc/1738/1

Published by the American Institute of Physics 


\title{
Application for Calculation of Mean Aerodynamic Chord of Arbitrary Wing Planform
}

\author{
Tomáš Vogeltanz \\ Tomas Bata University in Zlin, Faculty of Applied Informatics \\ Department of Informatics and Artificial Intelligence \\ nám. T.G. Masaryka 5555, 76001 Zlín, CZECH REPUBLIC
}

\begin{abstract}
This paper presents an application for the calculation of the mean aerodynamic chord (MAC) of an arbitrary wing planform. The MAC is most often used in the aerodynamic and stability analysis. The calculation uses a method where the MAC is defined by an array of chords. The MAC of each chord is computed as separated trapezoidal wing and then, the final MAC is calculated. This approach may find the accurate solution of complicated wing planforms, including elliptical, in the regime of low subsonic speeds. The first section describes the MAC and essential equations used for the computation. Finally, the application and two various examples of calculation are illustrated and discussed.
\end{abstract}

Keywords: aerodynamics; aircraft; calculation; MAC; mean aerodynamic chord; planform; software; subsonic; wing PACS: 07.05.Bx; 02.60.Jh; 07.05.Pj

\section{INTRODUCTION}

Various wing reference lengths are used in aerodynamic computations. One of the most important is the mean aerodynamic chord (MAC) which is an aerodynamic parameter referenced throughout aerodynamic and stability calculations in aircraft design. [1] [2]

It has been found both experimentally and theoretically that if the aerodynamic force is applied at a location $1 / 4$ chord back from the leading edge, the magnitude of the aerodynamic moment remains nearly constant with angle of attack on most low speed airfoils which is advantageous for the aerodynamic analysis. Engineers call the location where the aerodynamic moment remains constant the aerodynamic center (AC) of the airfoil. In contrast, for supersonic airfoils, the aerodynamic center is nearer the $1 / 2$ chord location. [3] [4]

For rectangular wings, the wing $\mathrm{AC}$ is the same as the airfoil AC. But for wings with some other planform (triangular, trapezoidal, compound, etc.) we have to find a mean aerodynamic center (MAC) which is the average for the whole wing. The computation of the MAC depends on the shape of the planform. [3] [5]

\section{MEAN AERODYNAMIC CHORD}

MAC is essentially an average chord of a lifting surface; in other words, it is the chord-weighted average chord length of the wing, defined as (1) where $S$ is a wing area, $b$ is a wing span, and $c$ is a chord length. MAC can be found for any lifting surfaces, including the horizontal and vertical stabilizers. [1] [2]

$$
M A C=\frac{2}{S} \cdot \int_{0}^{b / 2} c^{2} d y
$$

The computation of MAC is simple if the aircraft wing has a constant chord; however, this situation is almost never the case because it is often advantageous to introduce taper or sweep into the wing planform. For a linearly tapered (trapezoidal) wing, the equation (1) is equal to (2) where $\lambda$ is a taper ratio defined as (3), $c_{r}$ is a root chord, and $c_{t}$ is a tip chord. [2] [6] [1] [7] [5]

$$
M A C=\frac{2}{3} \cdot c_{r} \cdot\left(\frac{1+\lambda+\lambda^{2}}{1+\lambda}\right)
$$

International Conference of Numerical Analysis and Applied Mathematics 2015 (ICNAAM 2015)

AIP Conf. Proc. 1738, 120018-1-120018-4; doi: 10.1063/1.4951901

Published by AIP Publishing. 978-0-7354-1392-4/\$30.00 


$$
\lambda=\frac{c_{t}}{c_{r}}
$$

It is necessary to establish a representative average chord of the lifting surface and the location of this average chord longitudinally and laterally. The MAC distance from the center line (which means the distance on ' $y$ ' axis in most cases) may be calculated as (4) where $y_{r}$ is the $y$ coordinate of a root chord. [2] [6] [7]

$$
y_{M A C}=y_{r}+\frac{b}{2} \cdot \frac{1+2 \cdot \lambda}{3+3 \cdot \lambda}
$$

The location of the MAC leading edge on $x$ axis can be calculated as (5) where $x_{r}$ and $x_{t}$ are the $x$ coordinates of the leading edges of a wing root and wing tip. [7]

$$
x_{M A C_{L E}}=x_{r}+\left(x_{t}-x_{r}\right) \cdot \frac{1+2 \cdot \lambda}{3+3 \cdot \lambda}
$$

Then, the MAC coordinates might be specified as (6). [7] [3] [4]

$$
\left(x_{M A C}, y_{M A C}\right)=\left(x_{M A C_{L E}}+0.25 \cdot M A C, y_{M A C}\right)
$$

If the wing has multiple discrete panels of different taper, the integral definition should be applied or the equation (7) can be used once the MACs of the individual panels are known. [2] [4]

$$
M A C=\frac{\sum_{i=1}^{n} M A C_{i} \cdot S_{i}}{\sum_{i=1}^{n} S_{i}}=\frac{M A C_{1} \cdot S_{1}+M A C_{2} \cdot S_{2}+M A C_{3} \cdot S_{3}+\ldots+M A C_{n} \cdot S_{n}}{S_{1}+S_{2}+S_{3}+\ldots+S_{n}}
$$

The $x$ and $y$ coordinates of MAC can be found in the same manner presented in (7); of course, the $M A C$ has to be changed to $x_{M A C} L E$ or $y_{M A C}$, respectively. It should be noted that the number of MACs $=$ the number of panels -1 .

\section{APPLICATION SOFTWARE}

The application for the calculation of the mean aerodynamic chord of an arbitrary wing planform was developed under the Windows 7 Professional operating system; however, because the wxWidgets 3.0.2 library [8] was used for the implementation, the application may also run on Linux platform (e.g. Ubuntu). Moreover, the CodeLite 6.1.1 integrated development environment [9], $\mathrm{C}++$ programming language, and the MinGW 4.8.1 compiler [10] were used for the creation of this application. The graphical user interface may be seen in Figure 1.

The calculation and the input file format are based on the GETMAC console application released in [11]. A whole wing planform is defined by an array of chords and the calculation is separately performed on each trapezoidal wing segment defined by two chords.

Each chord is defined by its position of the leading edge on $x$ and $y$ axes, and its length. The unit is optional; it can be, for example, $1 \mathrm{~mm}$ per 1 pixel for small wing planforms. However, the $y$ coordinate must be specified from the smallest value to the biggest for the correct computation of MAC. Although the $y$ should be computed from the root chord (bottom), in this application, it is computed from the top of the image.

The user may parametrically define the chord information - simultaneously, the wing planform is drawn; furthermore, the canvas automatically customizes its size. The image of the wing planform may be saved as a PNG file. This format was chosen for its effective lossless compression [12] which is advantageous for this type of images, i.e. computer pictures with few colors. 


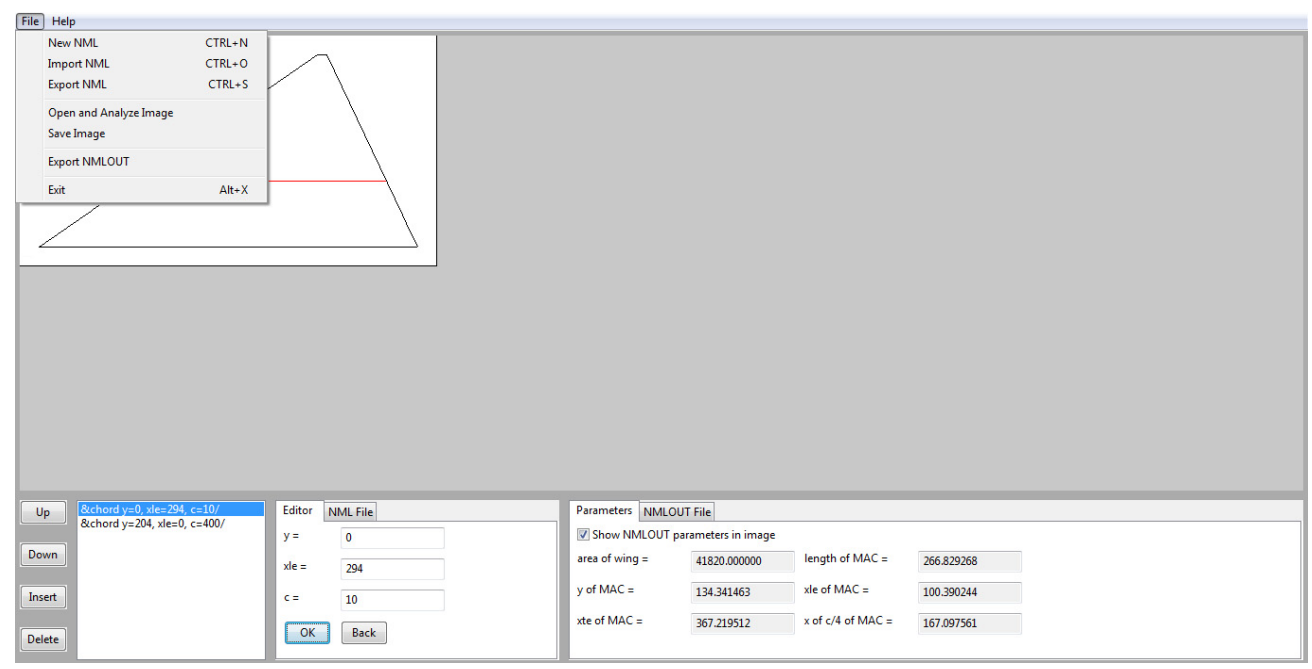

FIGURE 1. Graphical User Interface of Application for MAC Calculation

The application prints the area of wing, length of MAC, $y$ of MAC, $x_{L E}$ ( $x$ of Leading Edge) of MAC, $x_{T E}(x$ of Trailing Edge) of MAC, and $x$ of MAC. All output parameters are immediately recalculated when any change in specification of the wing occurs.

One special function of this application is the analysis of image with wing planform and its conversion to the parametric specification. The image has to be saved in PNG format with 2 colors - the white color defines the background and the black color specifies a planform. The algorithm simply reads the line by line and it searches for the black pixels; the first black pixel is the leading edge and the last black pixel is the trailing edge. This feature can help with the definition of an elliptical wing or with the analysis of any wing planform from a photograph.

\section{Calculation Experiments}

The following examples, which can be seen in Figure 2 and Figure 3, illustrate the accuracy of calculation. The first example (Figure 2) shows a wing planform of SAGITTA Demonstrator. The unit of $1 \mathrm{~mm} / \mathrm{px}$ was used for calculation and the results correspond with the values from the original research of the SAGITTA project in [13] where the wing reference area is $0.528 \mathrm{~m}^{2}$ and the mean aerodynamic chord is $0.667 \mathrm{~m}$. The small difference in wing areas is caused due to use of the integer type for the input values; the value of the second $y$ should be slightly higher, i.e. $1029.3333 \mathrm{~mm}$. It should be also noted, that $y$ of MAC was recalculated in order to describe the length from the root chord.

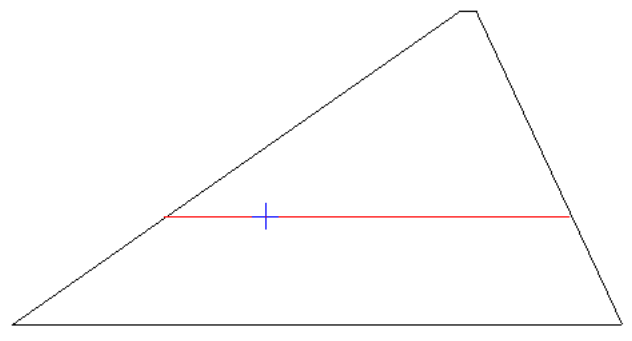

(a)

$$
\begin{aligned}
& \text { \&chord } y=0, x l e=1470, c=50 / \\
& \text { \&chord } y=1029, x l e=0, c=2000 /
\end{aligned}
$$

(b)

$\begin{array}{llll}\text { area of wing } & =527362.5 & \mathrm{~mm}^{2} \\ \text { length of MAC } & = & 667.0731705 & \mathrm{~mm} \\ \mathrm{y} \text { of MAC } & = & 175.682927 & \mathrm{~mm} \\ \text { xle of MAC } & = & 100.97561 & \mathrm{~mm} \\ \text { xte of MAC } & = & 918.0487805 & \mathrm{~mm} \\ \mathrm{x} \text { of MAC } & =417.7439025 & \mathrm{~mm}\end{array}$

(c)

FIGURE 2. Projection of SAGITTA wing planform with MAC (a), input file (b) and output parameters (c)

The second example in Figure 3 shows an elliptical wing, from [6], which was converted from a PNG file to a parametric representation by using the application. Before that, the PNG file was slightly edited and the number of colors was reduced to 2 (white and black). You can see that the line of the MAC is on the same position; moreover, the location of the MAC seems to be the same too. Consequently, this application has been validated for the MAC calculation of complicated planforms. 


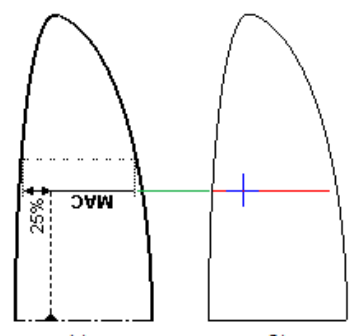

(a)

(b)

FIGURE 3. Projection of Elliptical Wing Planform with MAC; (a) original, (b) computed

\section{CONCLUSION}

This paper has presented the application software for the calculation of the mean aerodynamic chord of an arbitrary wing planform. First of all, the MAC and essential equations were described. In addition, the developed application and two various examples of calculation were illustrated.

It was proved that the MAC of complicated wing planforms may be computed by using the approximation of the multi-panel trapezoidal-wing calculation. However, some planforms, such as elliptical, are more difficult to define but they can be analyzed from an image file and converted to a parametric representation. Nevertheless, if an inappropriate scale is used, the real MAC may be slightly different for these types of planforms.

Because the results contain some very small differences (especially in wing area), the type of the values for the planform definition should be changed from integer to double. Furthermore, the use of a homogeneous material for building a wing is probably a necessary condition to achieving the calculated MAC. However, despite these little complications, the results are very accurate in comparison with the assumed values. As a result, this application may be reliably used for the MAC calculation of any planform with almost all subsonic airfoils.

\section{ACKNOWLEDGMENTS}

This work was supported by the Internal Grant Agency of Tomas Bata University in Zlín under the project No. IGA/FAI/2015/001.

\section{REFERENCES}

1. Ilan Kroo and Juan Alonso, "Wing Design", accessed: 2015, June 18, available: http://mail.tku.edu.tw/095980/6 Wing\%20Design.pdf?q=wing.

2. Paracreo, "Introduction to Aircraft Design: Mean Aerodynamic Chord", 2011, July 28, available: http://www.paracreo.com/resources_v2/articles/Paracreo\%20Aerodynamics\%20Article\%203\%20$\% 20$ Mean $\% 20$ Aerodynamic\%20Chord.pdf.

3. NASA, “Aerodynamic Center - ac", accessed: 2015, June 18, available: https://www.grc.nasa.gov/www/k12/airplane/ac.html.

4. Walter S. Diehl, The Mean Aerodynamic Chord and the Aerodynamic Center of a Tapered Wing, NACA Technical Report 751, 1942, Jan 1, pp. 413-422, available: http://ntrs.nasa.gov/archive/nasa/casi.ntrs.nasa.gov/19930091829.pdf.

5. David J. Peery, Aircraft Structures, Dover Publications, 1950, ISBN 978-0-486-48580-5.

6. Adam One, “Aerodynamics", 2013, December 23, available: http://adamone.rchomepage.com/index5.htm.

7. Ralph Carmichael, "getmac.f90”, 2013, April 18, available: http://www.pdas.com/packages/getmac.zip.

8. wxWidgets contributors, "wxWidgets: Cross-Platform GUI Library", 2015, available: http://wxWidgets.org.

9. CodeLite contributors, "CodeLite: A Free, open source, cross platform C,C++,PHP and JavaScript IDE", 2015, available: http://codelite.org.

10. MinGW contributors, "MinGW: Minimalist GNU for Windows", 2015, available: http://www.MinGW.org.

11. Ralph Carmichael, "Mean Aerodynamic Chord of a Wing”, 2013, February 13, available: http://www.pdas.com/getmac.html.

12. Tomáš Vogeltanz and Pavel Pokorný, "An Application for the Comparison of Lossless Still Image Compression Algorithms", International Journal of Circuits, Systems and Signal Processing, vol. 8, 2014, pp. 38-46, available: http://www.naun.org/main/NAUN/circuitssystemssignal/2014/a222005-086.pdf.

13. A. Hövelmann and C. Breitsamter, "Aerodynamic Characteristic of the SAGITTA Diamond Wing Demonstrator", Deutscher Luft- und Raumfahrtkongress, 2012, 14 p, available: http://mediatum.ub.tum.de/doc/1127549/file.pdf. 